\title{
Aspergillus fumigatus carrying TR34/L98H resistance allele causing complicated suppurative otitis media in Tanzania: Call for improved diagnosis of fungi in sub-Saharan Africa
}

\author{
Martha F. Mushi ${ }^{i^{*}}$, Gustave Buname ${ }^{2}$, Oliver Bader ${ }^{3}$, Uwe Groß $^{3}$ and Stephen E. Mshana ${ }^{1}$
}

\begin{abstract}
Background: Suppurative otitis media (SOM) is a major public health concern worldwide and is associated with increased morbidity. Cases of fungal suppurative otitis media were studied to establish the effect of fungi in otitis media.

Methods: Ear swabs from 410 patients were collected aseptically using sterile cotton swabs from discharging ear through perforated tympanic membrane. Swabs were subjected to microscopic and culture investigations. The species of fungal growing on Sabouraud's agar were identified using MALDI-TOF MS. For moulds broth micro dilution method following EUCAST guidelines was employed to determine susceptibility patterns against itraconazole, voriconazole and posaconazole.
\end{abstract}

Results: A total of 44 (10.74\%) cases with positive fungal culture growth were studied. The median age of patients with fungal infection was 29.5 (IQR 16-43) years. Of 44 patients; 35 (79.6\%) had pure growth of one type of fungal. Candida albicans was the most common fungus isolated $(n=13 ; 29.6 \%)$ followed by Aspergillus versicolor $(n=8 ; 18.2 \%)$. A total of 7 (15.9\%) patients had disease complication at time of enrollment; of them 6 (13.6\%) had hearing loss. On follow up 7 (15.9\%) had poor treatment outcome. All five Aspergillus fumigatus strains resistant itraconazole with reduced susceptibility to voriconazole and posaconazole carried carrying TR34/L98H resistance allele. In addition, all Penicillium citrinum isolates were resistant to voriconazole while all Penicillium sumatrense were resistant to both itraconazole and voriconazole. There were non-significant association of poor treatment outcome and female gender, being HIV positive and being infected with moulds.

Conclusion: Fungal infections play a significant role in SOM pathology in our setting. Diagnosis of fungal infections in developing countries should be improved so that appropriate management can be initiated on time to prevent associated complications.

Keywords: Suppurative otitis media, Moulds, Yeast, Diagnosis of fungi

Abbreviations: BMC, Bugando Medical Centre; CUHAS, Catholic University Health and Allied Sciences; EUCAST, European Committee on Antimicrobial Susceptibility Testing; HIV, Human Immunodeficiency Virus; IQR, Interquartile range; MALDI-TOF, Matrix-Assisted Laser Desorption Ionization-Time of Flight; SDA, Sabouraud's Dextrose Agar; SOM, Suppurative Otitis Media

\footnotetext{
* Correspondence: marthamushi@yahoo.com

${ }^{1}$ Department of Microbiology and Immunology, Weill Bugando School of

Medicine, Catholic University of Heath and Allied Sciences (CUHAS), P.O. BOX

1464, Mwanza, Tanzania

Full list of author information is available at the end of the article
} 


\section{Background}

Suppurative otitis media (SOM) is characterized by the inflammation of the middle ear and mastoid, tympanic membrane perforation as well as discharge [1]. The tympanic membrane perforation may result in increased exposure of the middle ear to pathogens [2, 3]. In developing countries, SOM is a major cause of preventable hearing loss [4-6], its incidence ranges from 7 to $46 \%$ and is common amongst children of lower socio-economic status [7, 8]. In Tanzania, SOM constitutes a major cause of otorhinolaryngology clinic visits and contributes significantly to high morbidity and long term hearing loss [9-11]. While bacterial pathogens (most prominently Pseudomonas aeruginosa and Staphylococcus aureus [12-15]) have commonly been documented as the cause of SOM in developing countries, the role of fungal infections among patients with SOM is still underestimated [3, 16, 17]. Fungal infections among patients with SOM account for $2.1-25 \%$ of cases [12-14] and Aspergillus spp. are the commonest cause accounting for $92.1 \%$ of cases $[12,18]$.

Fungal infections are mainly attributed by compromised immune status, prolonged antibiotic use and immune suppressive therapy $[19,20]$. This aspect of mycosis has gained importance over the recent years because of the excessive use of broad-spectrum antibiotics, and an increase in the number of immunodeficiency conditions such as immunoglobulin deficiency, malignant neoplasms, immunosuppressive therapy (corticosteroids and cytotoxic chemotherapy), diabetic mellitus as well as AIDS [3, 8, 21].

Fungi have been least documented as the cause of SOM in Africa probably due to lack of laboratory diagnose for fungal infections in these settings. Here, we determined the prevalence of fungal occurring in the otolaryngology clinic and surgical wards of Bugando Medical Centre (BMC), Mwanza Tanzania complicating cases of SOM.

\section{Methods}

\section{Sample collection}

A total of 410 patients with suppurative otitis media attending at $\mathrm{BMC}$ otorhinolaryngology clinic in a period of three months were investigated. For each patient with suppurative otitis media, ear swabs were aseptically collected using sterile cotton swabs (Heinz Herenz Hamburg, Germany).

\section{Microbiology}

Swabs were transported to microbiology laboratory using Stuart transport media (HiMedia, India) and subjected to microscopic investigation by Gram's stain as well as culture on sheep blood (BA), Mackonkey (MCA) and sabouraud's dextrose agar supplemented with $50 \mathrm{mg} / \mathrm{ml}$ gentamicin and $50 \mathrm{mg} / \mathrm{ml}$ chloramphenicol (SDA) (Oxoid, UK). Plates were aerobically incubated at $35{ }^{\circ} \mathrm{C}$ for 24-48 h. Fungal cultures with at least $2+$ growth were considered as significant [22].
Significant positive fungal pathogens on the SDA were further characterized while all other organisms were subjected to the normal procedures according to the BMC microbiology laboratory (Southern African Development Community Accreditation Service (SADCAS) with a unique number MED 002) standard operating procedures.

Growth on SDA plates was preliminary classified as mould or yeast based on the colony color and morphology. Yeast isolates were identified as growth of creamy to white colonies while moulds were identified as the filamentous colonies of various appearances [23]. CHROM agar (OXOID, England) was used as previously described for preliminary identifications of yeast in case of mixed growth [23].

Species identification was done by matrix-assisted laser desorption ionization-time of flight (MALDI-TOF) mass spectrometry (Bruker Daltonics, Bremen, Germany) on extracted cells harvested from agar plates (yeasts) or from overnight shaking cultures (moulds) in sabouraud's broth (Oxoid, Wesel, Germany) as previous described [24, 25].

All moulds isolated were subjected to antimicrobial susceptibility testing using broth micro-dilution method according to EUCAST guidelines [26]. The antifungal agents tested were itraconazole, voriconazole (Discovery Fine Chemicals, Bournemouth, United Kingdom) and posaconazole (MSD Sharp and Dohme, Haar, Germany). Plates were incubated at $37{ }^{\circ} \mathrm{C}$ for $48 \mathrm{~h}$ and MIC values for all drugs were visually determined as the lowest concentrations with no visible growth.

For azole resistant $A$. fumigatus isolates, the cyp $51 A$ locus was amplified by PCR and the resulting fragments sequenced as described before [27].

All patients were managed according to the standard protocol at BMC. All patients were initially treated conservatively, including aural toilet and applications of appropriate antibiotics as per bacterial culture and sensitivity results [15]. Whenever fungal infections were suspected, patients were treated empirically; this included removal of fungal debris and application of boric acid or combinations of antibiotics and antifungals. In the current study, poor treatment outcome was defined as persistence of otorrhea while the treatment success was defined as the disappearance of the signs and symptoms after 14 weeks follow-up [15].

\section{Results}

Out of 410 patients with SOM, 44 (10.7 \%, 95 \% CI 7.713.7) had significant fungal growth. The studied cases are summarized in Table 1 . The median age (inter quartile range (IQR) of patients with fungal infections was 29.5 (IQR 16-43) years. Male formed majority of patients with fungal infections $(n=24,54.6 \%)$. The median duration of illness was 18 (IQR 5-24) weeks. Of the 44 patients with fungal infections, 6 (13.6\%) were HIV positive. On Mann 
Table 1 Summary of 44 cases of SOM with fungal infection

\begin{tabular}{|c|c|c|c|c|c|}
\hline ID & ${ }^{\mathrm{a}}$ Duration (weeks) & HIV & Isolates & Complication & Treatment outcome \\
\hline ES025 & 60 & Neg & A. flavus & Hearing loss & Not Cured \\
\hline ES036 & 60 & Neg & A. flavus & None & Cured \\
\hline ES091 & 70 & Neg & A. flavus & None & Cured \\
\hline ES093 & 12 & Neg & A. flavus & None & Cured \\
\hline ES193 & 8 & Neg & A. flavus, A. versicolor & None & Not Cured \\
\hline ES026 & 4 & Neg & A. fumigatus & None & Cured \\
\hline ES028 & 32 & Neg & A. fumigatus & None & Cured \\
\hline ES095 & 20 & Neg & A. fumigatus & None & Cured \\
\hline ES027 & 90 & Pos & A. fumigatus, A. flavus & None & Cured \\
\hline ES057 & 18 & Neg & A. sydowii, P. chrysogenum & Mastoditis & Cured \\
\hline ES117 & 18 & Neg & A. versicolor & None & Cured \\
\hline ES130 & 32 & Neg & A. versicolor & Hearing loss & Cured \\
\hline ES143 & 18 & Neg & A. versicolor & Hearing loss & Cured \\
\hline ES179 & 18 & Neg & A. versicolor & None & Cured \\
\hline ES195 & 15 & Neg & A. versicolor, A. sydowii, P. sumatrense & Hearing loss & Cured \\
\hline ES112 & 24 & Neg & A. versicolor, P. citrinum & None & Not Cured \\
\hline ES001 & 24 & Neg & C. albicans & None & Cured \\
\hline ES002 & 12 & Neg & C. albicans & None & Cured \\
\hline ES016 & 2 & Neg & C. albicans & None & Cured \\
\hline ES030 & 12 & Neg & C. albicans & None & Cured \\
\hline ES032 & 48 & Neg & C. albicans & Hearing loss & Not Cured \\
\hline ES033 & 24 & Neg & C. albicans & None & Cured \\
\hline ES048 & 4 & Neg & C. albicans & None & Cured \\
\hline ES063 & 10 & Neg & C. albicans & None & Cured \\
\hline ES064 & 1 & Neg & C. albicans & None & Cured \\
\hline ES065 & 4 & Pos & C. albicans & None & Cured \\
\hline ES083 & 1 & Neg & C. albicans & None & Cured \\
\hline ES185 & 13 & Neg & C. albicans & None & Cured \\
\hline ES097 & 20 & Neg & C. albicans & None & Cured \\
\hline ES031 & 24 & Neg & C. parapsilosis & Hearing loss & Not Cured \\
\hline ES035 & 0.5 & Neg & C. parapsilosis & None & Cured \\
\hline ES061 & 6 & Neg & C. parapsilosis & None & Cured \\
\hline ES019 & 1 & Pos & C. tropicalis & None & Cured \\
\hline ESO20 & 1 & Pos & C. tropicalis & None & Cured \\
\hline ES037 & 1 & Neg & C. tropicalis & None & Cured \\
\hline ES052 & 72 & Neg & C. tropicalis & None & Cured \\
\hline ES058 & 20 & Neg & C. tropicalis & None & Cured \\
\hline ESO05 & 3 & Neg & C. tropicalis, A. fumigatus & None & Cured \\
\hline ES175 & 9 & Pos & P. chrysogenum & None & Cured \\
\hline ES077 & 18 & Neg & P. citrinum & None & Cured \\
\hline ES125 & 48 & Pos & P. citrinum & None & Not Cured \\
\hline ES085 & 6 & Neg & P. citrinum, A. versicolor & None & Not Cured \\
\hline ES132 & 24 & Neg & P. citrinum, P. lilacinus & None & Cured \\
\hline ES149 & 18 & Neg & P. sumatrense, A. versicolor & None & Cured \\
\hline
\end{tabular}

${ }^{a}$ Duration indicate the period the patients has stayed with otitis media before presenting at otolaryngology clinic of BMC 
Whitney ranksum test, there were non-significant difference in the median duration of illness between patients with fungal infections and those without fungal infections (18, IQR: $5-24$ vs. 12 IQR: $5-24, p=0.5749$ ).

Out of the 44 positive fungal cultures, 35 (79.6\%) produced pure growth of only one fungal species, while nine (20.4 \%) had mixed fungal growth. A total of 23 (52.3\%) patients were positive for moulds and $21(47.7 \%)$ positive for yeast. Candida albicans was the most commonly isolated fungal $(n=13,29.6 \%)$ followed by Aspergillus versicolor $(n=8,18.2 \%)$ (Table1). A total of seven (15.9\%) patients had disease complication at time of enrollment; of them six (13.6 \%) had hearing loss. On follow up, seven (15.9\%) had poor treatment outcome. Out of 366 patients with no fungal growth; $30(8.2 \%)$ had hearing loss as compared to $7 / 44$ (15.9 \%) of those with fungal infections $(p=0.0339)$.

All five strains of Aspergillus fumigatus isolates were resistant to itraconazole and showed reduced in vitro susceptibility to both voriconazole and posaconazole. Sequencing of the cyp $51 \mathrm{~A}$ locus revealed these isolates to carry the $\mathrm{TR}_{34} /$ L98H resistance allele. Penicillium citrinum isolates were resistant to voriconazole and had reduced susceptibility to itraconazole and posaconazole. All Penicillium sumatrense were resistant to both itraconazole and voriconazole, and showed reduced susceptibility to posaconazole (Table 2).

There were non- significant association of the poor treatment outcome with increase in age, female gender, being HIV positive and being infected with moulds Table 3.

\section{Discussion}

SOM is highly associated with lower social economic status, potentially due to poor hygiene, low access to medical care, or lack of knowledge [28]. In the current study, the majority of patients with positive fungal growth were from rural area where there are no hospitals with otolaryngology services. Most of these patients presented in BMC after six months of illness.

As seen in other studies [10], C. albicans and Aspergillus spp. were the most commonly isolated fungal from SOM specimens. The ability of $C$. albicans to maintain the synergistic relationship with bacterial pathogenic flora of skin like $S$. aureus and ecological niche of these isolates may explain the findings $[29,30]$.

Fungal have mainly been documented as opportunistic pathogens causing infections in immunocompromised patients. Among the 44 fungal-infected cases of SOM studied here, only six (15.9 \%) were HIV positive, which increased the risk of getting poor treatment outcome of SOM by 1.2 fold. The immunocompromised state of these patients could explain this observation. However, this observation was not statistically significant. This necessitates the need to identify other risk factors associated with fungal infections among HIV-negative individuals in future studies.
Table 2 Antimicrobial susceptibility patterns

\begin{tabular}{|c|c|c|c|c|}
\hline ID & Species & Itraconazole & Voriconazole & Posaconazole \\
\hline ES93A & Aspergillus flavus & 0.5 & 0.25 & 0.25 \\
\hline ES05B & Aspergillus fumigatus & 32 & 1 & 0.5 \\
\hline ES026 & Aspergillus fumigatus & 32 & 4 & 1 \\
\hline ES95 & Aspergillus fumigatus & 32 & 2 & 1 \\
\hline ES95 & Aspergillus fumigatus & 32 & 1 & 0.5 \\
\hline ES95 & Aspergillus fumigatus & 32 & 2 & 1 \\
\hline ES57A & Aspergillus sydowii & 0.5 & 0.5 & 0.25 \\
\hline ES95B & Aspergillus sydowii & 0.5 & 0.25 & 0.5 \\
\hline ES85B & Aspergillus versicolor & 0.25 & 0.03 & 0.25 \\
\hline ES93B & Aspergillus versicolor & 0.13 & 0.5 & 0.5 \\
\hline M112A & Aspergillus versicolor & 0.5 & 0.25 & 0.25 \\
\hline M117 & Aspergillus versicolor & 0.25 & 0.13 & 0.5 \\
\hline M130 & Aspergillus versicolor & 1 & 0.5 & 0.5 \\
\hline M143 & Aspergillus versicolor & 0.5 & 0.5 & 0.5 \\
\hline M149A & Aspergillus versicolor & 0.5 & 0.25 & 0.25 \\
\hline M179 & Aspergillus versicolor & 0.06 & 0.03 & 0.06 \\
\hline M132B & $\begin{array}{l}\text { Paecilomyces } \\
\text { lilacinus }\end{array}$ & 0.5 & 0.13 & 0.25 \\
\hline ES77 & Penicillium citrinum & 0.5 & 16 & 0.5 \\
\hline ES85A & Penicillium citrinum & 0.25 & 8 & 0.25 \\
\hline ES95A & Penicillium citrinum & 0.5 & 16 & 0.5 \\
\hline M112B & Penicillium citrinum & 0.5 & 16 & 0.25 \\
\hline M125 & Penicillium citrinum & 0.5 & 16 & 0.25 \\
\hline M132A & Penicillium citrinum & 0.5 & 16 & 0.25 \\
\hline ES95C & $\begin{array}{l}\text { Penicillium } \\
\text { sumatrense }\end{array}$ & 32 & 32 & 1 \\
\hline M149B & $\begin{array}{l}\text { Penicillium } \\
\text { sumatrense }\end{array}$ & 16 & 32 & 1 \\
\hline ES57B & $\begin{array}{l}\text { Penicillum } \\
\text { chrysogenum }\end{array}$ & 0.13 & 0.13 & 0.13 \\
\hline
\end{tabular}

In the current study, with eight isolates, $A$. versicolor was the predominant Aspergillus species. A. versicolor is a highly resilient/resistant fungus found in damp indoor environment, able to produce hepatotoxic and carcinogenic mycotoxin sterigmatocystin [31, 32].

Patients infected with moulds had 5.5 times higher risk of getting poor treatment outcome than patients with Candida infections. This could be explained by the fact that the majority (in fact all of the A. fumigatus, $P$. sumatrense, and $P$. citrinum isolates) were resistant to at least one commonly used antifungal agent. For the past two decades the increasing occurrence of $A$. fumigatus isolates resistant to common azoles antifungal agents has been described, including in Tanzania [33]. In our study, all $A$. fumigatus isolates indeed carried the $\mathrm{TR}_{34} / \mathrm{L} 98 \mathrm{H}$ allele, which shows that the environmental occurrence of these isolates is also clinically relevant in Tanzania. Additionally, 
Table 3 Factors associated with poor treatment outcome among patients SOM due to fungal infection

\begin{tabular}{|c|c|c|c|}
\hline Variable & $\begin{array}{l}\text { Poor treatment } \\
\text { outcome n (\%) }\end{array}$ & $\mathrm{OR}(95 \% \mathrm{Cl})$ & $P$ \\
\hline $\mathrm{Age}^{\mathrm{a}}$ & 29.5(IQR16-43) & $1.04(1.00-1.08)$ & 0.062 \\
\hline \multicolumn{4}{|l|}{ Sex } \\
\hline Male (24) & $3(12.50)$ & 1 & \\
\hline Female (20) & $4(20.00)$ & $1.75(0.34-8.95)$ & 0.5 \\
\hline \multicolumn{4}{|l|}{ HIV status } \\
\hline Negative (38) & $6(15.79)$ & 1 & \\
\hline Positive (6) & $1(16.67)$ & $1.07(0.11-10.82)$ & 0.95 \\
\hline |llness duration ${ }^{a}$ & 18(IQR 9-32) & $0.98(0.94-1.02)$ & 0.49 \\
\hline \multicolumn{4}{|c|}{ Fungal growth type } \\
\hline Yeast (21) & $2(9.52)$ & 1 & \\
\hline Molds (23) & $5(21.74)$ & $2.64(0.45-15.36)$ & 0.2 \\
\hline
\end{tabular}

Median

all isolates of $P$. citrinum and $P$. sumatrense were resistant to the lead antifungal agent itraconazole or showed reduced susceptibility to voriconazole. However in these species the underlying resistance mechanism is unknown.

In most clinical settings in resource-constrained countries like Tanzania where fungal diagnostics is underdeveloped; treatment of fungal infections relies solely on the empirical use of topical azole agents, which has previously been reported to be in effective for $A$. versicolor [34], the most frequent moulds isolated here and is probably similarly ineffective for azole-resistant $A$. fumigatus, a significant emerging problem.

In the current study $15.9 \%$ of patients infected with fungi developed complications, mostly irrevocable hearing loss, proving that hearing loss is a major complication developed by patients with suppurative otitis media [35]. The clinical impact of fungal infections in patients with SOM should drive the effort to improve fungal diagnostics in developing countries.

One of the limitations of this study is the possibility of skin flora contamination during sample collections. However; the quantification of fungal growth significantly minimized the chances suggesting that majority of patients had real fungal infections.

\section{Conclusion}

Fungal infections played a significant role in SOM pathology under low resource settings. Diagnosis of fungal infections in developing countries should be improved so that appropriate management can be initiated on time to prevent associated complications.

\section{Acknowledgement}

The authors would like to thank Agnieszka Goretzki, Eliaingiringa Moshi, Dr. Alfred Mwalutende and Emilia Gómez for expert technical assistance.

\section{Funding}

This work was supported by research grant from CUHAS to MFM and the Institute for Medical Microbiology, University Medical Center Goettingen, Germany.

Availability of data and materials

All data support the findings is contained in the manuscript.

\section{Authors' contributions}

MFM, SEM, GB and UG designed the study. GB did sample collections. MFM and $O B$ did laboratory work. MFM, OB and SEM participate in data analysis. MFM, OB, UG and SEM wrote the manuscript. All authors revised the final version of manuscript. All authors read and approved the final manuscript.

\section{Competing interests}

The authors declare that they have no competing interests.

\section{Consent for publication}

Not applicable.

\section{Ethics approval and consent to participate}

Ethical clearance for conducting this study was granted by joint CUHAS/BMC research ethics and review committee. Permission to conduct the study was sought from otolaryngology clinic and surgical wards of BMC and all patients were requested to sign the informed consent form before recruitment. All patients' data were confidential.

\section{Author details}

${ }^{1}$ Department of Microbiology and Immunology, Weill Bugando School of Medicine, Catholic University of Heath and Allied Sciences (CUHAS), P.O. BOX 1464, Mwanza, Tanzania. ${ }^{2}$ Department of Surgery, Bugando Medical Center, Mwanza, Tanzania. ${ }^{3}$ Institute of Medical Microbiology, University Medical Center Goettingen, Göttingen, Germany.

Received: 22 October 2015 Accepted: 23 August 2016

Published online: 02 September 2016

\section{References}

1. Giebink GS. The microbiology of otitis media. Pediatr Infect Dis J. 1989;8(1):S21.

2. Miró N. Controlled multicenter study on chronic suppurative otitis media treated with topical applications of ciprofloxacin $0.2 \%$ solution in singledose containers or combination of polymyxin $B$, neomycin, and hydrocortisone suspension. Otolaryngol Head Neck Surg. 2000;123(5):617-23.

3. WHO. Child and adolescent health and development, prevention of blindness and deafness. Chronic suppurative otitis media Burden of Illness and management options. 2004. ISBN 92-4-159158 7

4. Alabbasi AM, Alsaimary IE, Najim JM. Prevalence and patterns of chronic suppurative otitis media and hearing impairment in Basrah city. Afr J Med Med Sci. 2010;1(4):129-33.

5. Bakari AA, Adoga AA, Afolabi OA, Kodiya AM, Ahmad BM. Pattern of chronic suppurative otitis media at the national Ear care centre Kaduna, Nigeria. J Med Trop. 2010;12:22-5.

6. Manni JJ, Lema PN. Otitis media in Dar es Salaam, Tanzania. J Laryngol Otol. 1987;101:222-8.

7. Iqbal K, Khan MI, Satti L. Microbiology of chronic otitis media: experience at dera Ismail Khan. Gomal J Med Sci. 2011;9(2):189-93.

8. Kumar H, Seth S. Bacterial and fungal study of 100 cases of chronic suppurative otitis media. J Clin Diagn Res. 2011;5(6):1224-7.

9. Aquino JD, Pereira SH, Aquino JNPD, Neto RG, Carvalho MR, Filho NAC. Bacterial Findings Found in the Chronic Otitis Media Secretion: Comparative Study Between Cholesteatoma (OMCC) and Simple Chronic Otitis Media (SCOM). International Arch Otorhinolaryngol. 2009;13(3):287-92.

10. Sanjana RK, Singh Yl, Reddy NS. Aerobic bacteriology of Chronic Suppurative Otitis Media in a tertiary care hospital, A retrospective study. J Coll Med Sci Nepal. 2011;7(2):1-8.

11. Moshi NH, Minja BM, Ole-Lengine L, Mwakagile DS. Bacteriology of chronic otitis media in Dar es Salaam, Tanzania. East Afr Med J. 2000;77(1):20-2.

12. Ibekwe $A O$, al Shareef $Z$, Benayam $A$. Anaerobes and fungi in chronic suppurative otitis media. Ann Otol Rhinol Laryngol. 1997;106(8):649-52.

13. Loy A, Tan A, Lu P. Microbiology of chronic suppurative otitis media in Singapore. Singap Med J. 2002;43(6):296-9. 
14. Aslam MA, Ahmed Z, Azim R. Microbiology and drug sensitivity patterns of chronic suppurative otitis media. J Coll Physicians Surg Pak. 2004;14(8):459-61.

15. Mushi MF, Mwalutende AE, Gilyoma JM, Chalya PL, Seni J, Mirambo MM, Mshana SE. Predictors of disease complications and treatment outcome among patients with chronic suppurative otitis media attending a tertiary hospital, Mwanza Tanzania. BMC Ear Nose Throat Disord. 2016;16(1):1.

16. Pelikan Z. Chronic Otitis Media (Secretory) and nasal allergy. Scriptamedica (BRNO). 2006;79(4):177-98.

17. Singh $A H$, Basu R, Venkatesh A. Aerobic bacteriology of chronic suppurative otitis media in Rajahmundry, India. Biol Med. 2012;4(2):73-9.

18. Talwar P, Chakrabarti A, Kaur P, Pahwa R, Mittal A, Mehra Y. Fungal infections of ear with special reference to chronic suppurative otitis media. Mycopathologia. 1988;104(1):47-50.

19. Alo MN, Anyim C, Okonkwo EC, Orji JO. Prevalence, Antibiogram of Bacterial Pathogens Associated with Otitis Media among Primary School Children in Ebonyi State. Int J Pharm Bio Sci. 2012;1:2278-3008.

20. Lodhi M, Munir T, Aziz K, Lodhi H. Chronic suppurative otitis media, empiric quinolones in children. Prof Med J. 2010;17(3):420-4.

21. Probst R, Grevers G, Iro H, editors. Basic Otorhinolaryngology, a step by step learning guide. 2nd ed. 2006.

22. Winn WC, Koneman EW. Koneman's color atlas and textbook of diagnostic microbiology, 15th edn. Chicago: Lippincott williams \& wilkins; 2006.

23. Mushi MF, Mtemisika Cl, Bader O, Bii C, Mirambo MM, Groß U, Mshana SE. High Oral Carriage of Non-albicans Candida spp. among HIV-infected individuals. Int J Infect Dis. 2016;49:185-8.

24. Wieser A, Schneider L, Jung J, Schubert S. MALDI-TOF MS in microbiological diagnostics - identification of microorganisms and beyond (mini review) Appl Microbiol Biotechnol. 2012;93(3):965-74.

25. Bader O, Weig M, Taverne-Ghadwal L, Lugert R, Gross U, Kuhns M. Improved clinical laboratory identification of human pathogenic yeasts by matrixassisted laser desorption ionization time-of-flight mass spectrometry. Clin Microbiol Infect. 2011;17(9):1359-65.

26. Lass-Flörl C, Cuenca-Estrella M, Denning D, Rodriguez-Tudela J. Antifungal susceptibility testing in Aspergillus spp. according to EUCAST methodology. Med Mycol. 2006:44(sup1):319-25.

27. Bader O, Weig M, Reichard U, Lugert R, Kuhns M, Christner M, Held J, Peter S, Schumacher U, Buchheidt D. cyp51A-based mechanisms of Aspergillus fumigatus azole drug resistance present in clinical samples from Germany. Antimicrob Agents Chemother. 2013:57(8):3513-7.

28. Mohan U, Jindal N, Amritsar. 14: Fungal and Bacterial Flora of Chronic Suppurative Otitis Media in Amritsar (Punjab). IJO \& HNS. 1998;SO(2):175-7.

29. Tiwari S, Singh S, Jain S. Chronic bilateral suppurative otitis media caused by Aspergillus terreus. Mycoses. 1995;38(7-8):297-300.

30. MacFarlane TW, Samaranayake L. Ecology and epidemiology of Candida. Oral candidosis. Kent: Butterworth \& Co; 1990. p. 21-46

31. Bjurman J, Kristensson J. Volatile production by Aspergillus versicolor as a possible cause of odor in houses affected by fungi. Mycopathologia. 1992:118(3):173-8.

32. Engelhart S, Loock A, Skutlarek D, Sagunski $H$, Lommel A, Färber $H$, Exner M. Occurrence of toxigenic Aspergillus versicolor isolates and sterigmatocystin in carpet dust from damp indoor environments. Appl Environ Microbiol. 2002;68(8):3886-90.

33. Chowdhary A, Sharma C, van den Boom M, Yntema JB, Hagen F, Verweij PE, Meis JF. Multi-azole-resistant Aspergillus fumigatus in the environment in Tanzania. J Antimicrob Chemother. 2014;69(11):2979-83.

34. Gupta AK, Paquet M. Management of Onychomycosis in Canada in 2014. J Cutan Med Surg. 2015;19(3):260-73. doi:10.2310/7750.2014.14090.

35. Papp Z, Rezes S, Jókay I, Sziklai I. Sensorineural hearing loss in chronic otitis media. Otol Neurotol. 2003:24(2):141-4.

\section{Submit your next manuscript to BioMed Central and we will help you at every step:}

- We accept pre-submission inquiries

- Our selector tool helps you to find the most relevant journal

- We provide round the clock customer support

- Convenient online submission

- Thorough peer review

- Inclusion in PubMed and all major indexing services

- Maximum visibility for your research

Submit your manuscript at www.biomedcentral.com/submit
Biomed Central 